\title{
Analysis of Wind Heating System to Solve Winter Heating in Northeast China
}

\author{
Shi-wei Su \\ North China Electric Power University, Baoding 071003, China \\ 2446567415@qq.com
}

\begin{abstract}
Combined with the winter wind conditions in northeast China, three schemes are proposed to solve the problem of winter heating in the region. The three schemes of wind energy conversion directly into heat energy, wind energy compressed air heating and wind power heating are described, and the performance of the three schemes is explained. The relevant provisions of the northeastern region of housing heat calculation, at the same time get the corresponding program capital investment and recovery.
\end{abstract}

Keywords: wind power heating, northeastern district, squeeze, stir.

\section{Introduction}

Northeast winter time is long, low temperature. The heating value of straw is low and the demand is high, so straw heating seriously affect people's quality of life. The cost of coal heating is high and the pollution is high, and it is easy to cause carbon monoxide poisoning. Need to find a new heating method to solve the winter heating in rural areas in Northeast China. Japan, the United States and Europe in order to make more effective use of wind energy, and actively carry out the work of wind heating and initial success ${ }^{[1]}$, China's wind heating research and utilization is still relatively small. At present, China Agricultural University, the focus of the study of wind heating liquid mixing and heating, Shenyang University of Technology and Xi'an Jiaotong University research focused on liquid extrusion heating ${ }^{[2]}$.

In order to solve the problem of heating in winter, several concrete wind-heating schemes are used to solve the practical conditions in Northeast China. The feasibility and economy of the system are analyzed, which provides a theoretical basis for the practical application of wind power heating.

\section{Wind Distribution in Northeastern China}

China's vast territory, terrain differences, wind conditions in different parts of different, so our country is divided into wind-poor areas, wind general area, wind-rich area. According to China's wind power density distribution map released by the China Meteorological Administration, we can see that the northeastern region is located in the wind-rich area, and the total wind speed above $3.5 \mathrm{~m} / \mathrm{s}$ is more than $4000 \mathrm{~h}$. From the northeastern provinces to Xinjiang and other areas near the $2000 \mathrm{~km}$ wide wind belt is rich in the northern region, the wind speed is very large and smooth, wind power density 200-300W $/ \mathrm{m}^{2}$ above. These areas have reserves of 200 million 
kilowatts of wind resources can be developed, accounting for about $79 \%$ of the national reserves. The area is rich in wind energy resources, prevailing wind direction is stable, flat terrain, convenient transportation, engineering geological conditions, for the wind to provide an environmental basis for heating. ${ }^{[3]}$

\section{3 wind heating scheme and performance analysis}

\subsection{Wind energy directly into heat}

Liquid extrusion heating is the use of hydraulic pumps and damping holes for heating ${ }^{[4]}$. The wind turbine output shaft drives the hydraulic pump to rotate, so that the hydraulic oil from the narrow orifice high-speed discharge, high-speed jet of oil and wake in the low-speed oil phase impact. High-speed oil through the damping hole, due to molecular interaction with each other, friction and accelerate the molecular motion, the kinetic energy of oil into heat, oil temperature rise.

Liquid mixing and heating is connected with a stirring rotor on the rotating shaft of the wind wheel. The rotor is equipped with blades, and the stirring rotor is placed in a stirring tank full of liquid. The inner wall of the tank is a stator and is also equipped with blades ${ }^{[5]}$. When the wind wheel drives the rotor blades to rotate, the rotor stirs the liquid, the stator blades and the vessel wall as a vortex between the movement and continue to impact, friction, the mechanical energy into heat to improve the liquid temperature to get the required heat ${ }^{[6]}$.

Liquid squeeze heating is the result of the impact and friction between the liquid, it will not wear, burn and other problems damage the heating device, therefore, the reliability of the liquid extrusion heating. Squeezing heating and stirring heating are directly into the mechanical energy into heat, due to the lack of electrical energy conversion aspects of the wind to heat the conversion efficiency can reach $40 \%{ }^{[2]}$, compared to wind power efficiency is higher.

Stir heating device easy to manufacture, no wear parts, no strict requirements on the heat medium. Throughout the work process, all the input energy into heat, can be very good with the wind turbine output power characteristics to match the power factor.

Northeast winter minimum temperature of minus 40 degrees, so the performance of the liquid have a greater demand, and the whole set of equipment on the outdoors, the need for its thermal insulation measures, the construction of the accumulator to increase the area of the entire equipment and investment costs .

Direct use of wind energy makes the system more affected by the wind, for the unstable wind will make the system heating effect is greatly affected.

\section{2 wind heat pump heating}

The wind turbine turns compressed air, which is converted into heat, which is usually achieved by heat pumps. Wind turbine and AC motor drive the compressor through the transmission mechanism of the wind heat pump system. In the system, the wind turbine and the AC motor are connected by the transmission mechanism and the compressor. Under the control of the converter, the compressor can be driven by the wind or by the motor when there is no wind and the wind is insufficient ${ }^{[2]}$. 
The current heat pump performance coefficient COP between 2 to $3^{[7]}$, so the heat pump is an efficient heating device. However, due to the limitation of existing wind turbine output control ability, wind power output is difficult to control after wind power intensity change. It is not easy to realize real-time control of temperature in the room after heat pump compressor cooperation. When the transmission system adopts gearbox transmission, More complex, it is difficult to achieve the heat pump compressor power input structure of the integration with the connection; Northeast winter temperature is too low will cause heat exchanger frosting phenomenon. Frost layer to increase the thermal resistance, while blocking the fan channel caused by fan air volume decline, resulting in deterioration of heat pump performance ${ }^{[8]}$. Therefore, the implementation of heating in the northeastern region there are many restrictions.

\section{3 wind power heating}

Wind power generation is about to wind energy into electrical energy through the resistance wire into heat energy, wind power generating units, including wind turbine, transmission, generators, automatic control devices and supporting tower, wind turbine rotation will wind energy into mechanical energy, mechanical energy through the generator Into electrical energy, through the rectifier to stabilize the output voltage, the current waveform high degree of sinusoidal ${ }^{[9]}$, the current flows through the resistance of Joule's law we can see all the energy can be converted to heat heating.

When the output power of the wind turbine is not enough to satisfy the normal heating, the low power electric energy output by the wind turbine can be stored in the accumulator battery when the controller automatically switches to the power grid. The wind power generator can be used in the wind power controller, When the wind turbine output power is sufficient, it will automatically convert the use of wind turbine power to maintain normal heating. So the system is relatively small by the wind, the system structure is simple, the system almost no mechanical wear and tear, long service life.

\section{Economy Analysis of Wind Heating}

\section{1 initial cost of the program}

The wind turbine system includes wind turbine, transmission mechanism, heating equipment, heat storage equipment and so on. Compared to the wind power system, the wind power system includes the wind turbine, the transmission mechanism, the generator, the automatic control device and the supporting tower. The unit saves the generator, the automatic control device in the velocity modulation device, the brake installment and the grid needs the rectifier, the transformer and so on, altogether accounts for the wind turbine total price 25\%; the wind energy compressed air heating needs purchase additional heat pumps.

If the use of wind power heating method, you need to use $2000 \mathrm{~W}$ of wind turbines, wind turbines in accordance with the market price of the price of 4,500 yuan, coupled with thermal resistance, wind power controller, battery, support the total price of about 6,000 . If the use of wind heating, the required cost is about 4,500 yuan, but due 
to mechanical wear will shorten its service life, wind compressed air heating will need about 9,000 yuan.

\subsection{Investment recovery}

According to the investigation, we can see that the heating period (November to next April) is about 3 tons, and the current coal price is 800 tons per ton. Therefore, the annual coal-fired heating system is used in the rural areas of Northeast China. Of the cost of 2400 yuan, according to the pre-calculated investment costs, in 3 years (heat pump needs 5 years) can be recovered within the cost.

\section{Conclusions}

The use of wind power heating to solve the winter heating in northeast China can be a good solution to this problem, improve people's quality of life, and convenient, long life, once and for all, saving economic costs for the environment-friendly society.

\section{References}

[1] Wang Yuan-kai.Foreign wind-induced heat status abroad [J]. Solar Energy, 1987,03: 11-13.

[2] Zhao Haibo, Wu Kun.Heat-driven heat pump air conditioning system [J]. Building Energy \& Air-conditioning, 2010,01: 32-35 + 31.

[3] Song Jing. Wind power distribution and wind power planning in China [D]. North China Electric Power University, 2013.

[4] Li Huashan, Feng Xiaodong, Liu Tong. Research progress of wind power heating technology in China [J]. Solar Energy, 2008,09: 37-40.

[5] LIU Yang, HU Yi-huai.Parameter design of agitated wind-driven heating device [J] .Acta Solara Sinica, 2014,10: 1977-1980.

[6] Li Guofei, Zhang Hong.Study on Application of Stirred Wind Heating System [J]. Journal of Weifang University, 2004,06: 83-85.

[7] YANG Ling-yan, XU Wei, ZHU Qing-yu, XIAO Long.Analysis of development trend of international heat pump technology [J]., 2012,08: 1-8.

[8] Qiao Fengjie, Xu Yan. Development and problems of heat pump technology [J]. Information Technology 2011,02: 119-121.

[9] Liu Yu. Multiple small wind power grid system solutions [A]. Small and medium wind energy equipment and applications, 2016: 3. 